\title{
ACTUALIZACIÓN DE LOS INSTRUMENTOS DE EVALUACIÓN DE LAS PRÁCTICAS ESCOLARES: COORDINACIÓN ENTRE SERVICIOS PROVINCIALES, SERVICIOS DE INSPECCIÓN Y FACULTADES DE HUESCA Y TERUEL
}

School practice instruments of evaluation update: coordination between provincial services, inspection services and universities of Huesca and Teruel

Atualização dos instrumentos de avaliação das práticas escolares: coordenação entre serviços provinciais, serviços de inspecção e faculdadesde Huesca e Teruel

\author{
Alberto Aibar Solana (1) \\ Rosario Marta Ramo Garzarán (2) \\ Sandra Vázquez Toledo (1) \\ David Pérez Castejón (2) \\ Marta Liesa Orús (1) \\ Alberto Abarca Sos (2)
}

Financiación del trabajo: Universidad de Zaragoza. PIET_18_457.

(1) Facultad de Ciencias Humanas y de la Educación. Universidad de Zaragoza, España. Teléfono: +34 974239341. Correo electrónico: aibar@unizar.es

(2) Facultad de Ciencias Sociales y Humanas. Universidad de Zaragoza

\section{Resumen}

Dado que las prácticas escolares son un componente fundamental en la formación inicial del profesorado, el objetivo de esta propuesta fue el de revisar, reflexionar y actualizar los documentos e instrumentos de evaluación y calificación de las Prácticas Escolares I, II y III en los Grados de Magisterio en Educación Infantil y Magisterio en Educación Primaria. Un grupo de trabajo interprovincial (Huesca-Teruel) de diferentes agentes educativos (administración e inspección educativa, maestros tutores, coordinadores de prácticas de la universidad y profesores universitarios) desarrolló el trabajo durante el curso 18-19. Se siguió un proceso democrático y participativo con los diferentes agentes de la comunidad educativa que derivó en la construcción de nuevas herramientas de evaluación para los tres periodos de prácticas escolares generalistas que serán aplicadas y evaluadas durante el curso 19-20.

Palabras clave: Prácticum; prácticas escolares; evaluación; coordinación

Actualización de los instrumentos de evaluación de las prácticas escolares: coordinación entre servicios provinciales, servicios de inspección y facultades de Huesca y Teruel 


\begin{abstract}
Given that school training periods are fundamental components of the initial teacher training formation, the objective of this proposal was to review, reflect and update the documents and instruments for the evaluation and qualification of School Training Periods I, II and III at the Degrees of Teaching in Infant Education and Teaching in Primary Education. An inter-provincial working group (Huesca-Teruel) of different educational agents (administration and educational inspection, teacher tutors, coordinators from the University and university professors) developed the work during the academic course 18-19. A democratic and participative process was followed with different agents of the educational community. This process led to the construction of new assessment tools for the three periods of generalist school practices. These tools will be applied and evaluated during the 19-20 academic course.
\end{abstract}

Keywords: Practicum, school practices, evaluation, coordination

\title{
Resumo
}

Uma vez que as práticas escolares são um componente fundamental na formação inicial de professores, o objetivo desta proposta foi rever, refletir e atualizar os documentos e instrumentos de avaliação e classificação das Práticas Escolares I, II e III nas Licenciaturas de Formação de Professores da Educação Pré-Escolar e EducaçãoPrimária. Um grupo de trabalho inter-provincial (Huesca-Teruel) com diferentes agentes educativos (administração e inspeção educativa, professores tutores, coordenadores de práticas universitárias e professores universitários) desenvolveu o trabalho durante o curso 18-19. Seguiu-se um processo democrático e participativo com os diferentes agentes da comunidade educativa que levou à construção de novas ferramentas de avaliação para os três períodos de práticas escolares gerais que serão aplicadas e avaliadas durante o curso 19-20.

Palavras-chave: Práticas; práticas escolares; avaliação; coordenação

\section{Introducción}

Dentro de la formación inicial de los maestros, tanto de Infantil como de Primaria, las prácticas escolares constituyen asignaturas complejas, que exigen una gestión específica 
que requiere la coordinación e implicación de un gran número de centros y agentes (Monn†, Gonz«lez, Say\% y Trib\%,2003; p.129).

Se han descrito una serie de deficiencias en el proceso de tutorización de las prácticas escolares tales como la falta de coordinación entre escuela y universidad, una falta de claridad en las tareas a desarrollar por el alumnado o una serie de problemas con los instrumentos utilizados para la evaluación de su desempeño docente (Porto \& Bolarín, 2013). Considerando que la correcta organización del prácticum influye de manera decisiva en la percepción de la formación inicial recibida por los futuros maestros (Mendoza-Lira \& Covarrubias-Aplablaza, 2014), y que la evaluación y calificación del periodo de prácticas escolares resultan elementos fundamentales, parece necesario abordar una revisión pormenorizada de los instrumentos de evaluación con el fin de otorgar mayor calidad al proceso de formación inicial. Valorando la naturaleza multidimensional e interrelacionada de las necesidades que afectan al alumnado y a su educación, parece necesario abordar esa revisión mediante una respuesta coordinada y comprensiva por parte de múltiples agentes educativos (Nieto \& Portela, 2001). Generar un clima de colaboración entre todos los agentes influyentes puede y debe aportar importantes y sustanciales beneficios para la formación del alumnado y por ende para la comunidad educativa en su conjunto (Porto \& Bolarín, 2013).

El objetivo de esta propuesta fue el de revisar, reflexionar y actualizar los documentos e instrumentos de evaluación y calificación de las prácticas generalistas en los Grados de Magisterio en Educación Infantil y Magisterio en Educación Primaria.

\section{Contextualización}

Esta experiencia se sitúa en dos centros de la Universidad de Zaragoza, la Facultad de Ciencias Sociales y Humanas en Teruel y la Facultad de Ciencias Humanas y de la Educación en Huesca. El proceso de revisión se ha circunscrito a las asignaturas de Prácticas Escolares generalistas (Prácticas escolares I, II y III) tanto en el Grado de Maestro en Educación Infantil como en Primaria. Estas tres asignaturas tienen 6, 14 y 10 créditos y se desarrollan en el segundo, tercer y cuarto curso respectivamente. Esta experiencia tiene como público objetivo final a todo el alumnado participante en esos periodos de prácticas, un total de 1000 alumnos.

En base a la literatura existente (Monnł et al., 2003; Nieto \& Portela, 2001) y a un proceso de reflexión previo de los diferentes agentes de la comunidad educativa 
(maestros tutores de los centros educativos, coordinadores de prácticas de la universidad, profesores de la universidad, cuerpo de inspección y administración educativa pública) en el que se concluía la necesidad de mejora de los procesos y procedimientos de prácticas como elemento fundamental en la formación inicial del profesorado, se constituyó un grupo de trabajo inter-provincial constituido por diferentes agentes educativos para llevar a cabo ese proceso de análisis y modificación de los documentos e instrumentos de evaluación y calificación de las prácticas generalistas.

\section{Diseño y desarrollo}

El proceso de revisión de las asignaturas de prácticas escolares se muestra en la Tabla 1.

Tabla 1.

Proceso de revisión de las Prácticas Escolares.

\begin{tabular}{|c|c|c|}
\hline Acciones & Temporalización & $\begin{array}{l}\text { Responsables } \\
\text { implicados }\end{array}$ \\
\hline $\begin{array}{l}\text {--Reuniones iniciales. } \\
\text {.-Elaboración documento con competencias, documentos específicos a } \\
\text { revisar, instrumentos de seguimiento y evaluación. } \\
\text {.-Formación conjunta COFOS }{ }^{1} \text {, coordinadores y cuerpo de inspección }\end{array}$ & $\begin{array}{l}\text { Septiembre } 2018 \\
\text { Octubre } 2018\end{array}$ & $\begin{array}{l}\text { Grupos de } \\
\text { trabajo } \\
\text { COFOS }\end{array}$ \\
\hline $\begin{array}{l}\text {-Grupos de discusión por provincia: detección de necesidades y de } \\
\text { aspectos a mejorar. }\end{array}$ & Octubre 2018 & $\begin{array}{l}\text { Grupos de } \\
\text { trabajo }\end{array}$ \\
\hline .-Coordinación Huesca - Teruel & $\begin{array}{c}\text { Octubre } 2018, \\
\text { febrero y abril } 2019\end{array}$ & Coordinadores \\
\hline $\begin{array}{l}\text { - Cuestionario on-line dirigido a agentes educativos. } \\
\text {--Sesiones de trabajo para el análisis, reflexión y modificación de los } \\
\text { documentos. } \\
\text {.-Utilización de las planillas de evaluación de los funcionarios en prácticas } \\
\text { como referencia. }\end{array}$ & $\begin{array}{l}\text { Noviembre } 2018 \\
\quad \text { enero } 2019\end{array}$ & $\begin{array}{l}\text { Grupos de } \\
\text { trabajo }\end{array}$ \\
\hline -Adopción de acuerdos en aspectos controvertidos. & Marzo 2019 & Coordinadores \\
\hline $\begin{array}{l}\text {-Revisión de la documentación por agentes educativos ajenos al proceso } \\
\text { pero expertos colaboradores en la temática. }\end{array}$ & Mayo 2019 & Maestros tutores \\
\hline $\begin{array}{l}\text {-Sesión final conjunta. Adopción de acuerdos y presentación de } \\
\text { documentos finales. } \\
\text {.-Proceso de evaluación y seguimiento para el curso 19-20. }\end{array}$ & Junio 2019 & Grupo de trabajo \\
\hline
\end{tabular}

Notas: COFOS: Coordinadores de Formación en los centros educativos de Aragón.

\section{Evaluación}

La evaluación de los instrumentos generados se establece para el curso 19-20. Se aplicarán en ambas provincias con ligeras diferencias en su estructura. Por ejemplo, el criterio de calificación va a ser diferente en algún apartado (Sí-No vs. Sí- A veces - 
No). Todos estos elementos se evaluarán y, en base a criterios de calidad educativa y de mejor funcionamiento en el proceso de aplicación, se acabará por establecer una herramienta común. La evaluación se realizará a partir de formularios on-line y de grupos de discusión que se realizarán independientemente en cada una de las provincias con los maestros que van a aplicar dichas herramientas. En base a los resultados aportados se realizará un proceso de revisión de los instrumentos entre los diferentes agentes de la comunidad educativa implicada para consensuar una herramienta definitiva. Cabe destacar que, tal y como se hizo en el proceso de construcción de los instrumentos inicialmente generados, su estructura y categorización tendrá como referencia las herramientas de evaluación de los maestros funcionarios de prácticas del Gobierno de Aragón.

\section{Conclusiones}

Con esta experiencia de trabajo cooperativo se ha pretendido mejorar y actualizar las herramientas de evaluación que se utilizan para las prácticas escolares en los grados de Magisterio en Educación Infantil y Educación Primaria. Confiamos en que esta experiencia fomente unos mayores niveles de coordinación entre la universidad, los servicios provinciales de educación y de inspección y los maestros tutores de los centros.

Esta experiencia es una oportunidad única de mejorar el proceso de tutorización y evaluación de las prácticas escolares. Constituye un avance hacia procesos de tutorización serios, rigurosos y de calidad avalados por la comunidad educativa.

\section{Referencias}

Mendoza-Lira, M., \& Covarrubias-Apablaza, C.G. (2014). Valoración del prácticum de los grados del magisterio desde la perspectiva de sus estudiantes. Revista Electrónica Educare, 18(3), 111-142.

Monne, P., Gonz«lez, R., Say\%, R., \& Trib\%o(2003). El Practicum en los t,tulos de maestro de la Universidad de Barcelona. Iglesias, M., Zabalza, M, Cid, A. \& Raposo, M. (coords.). El Practicum como compromiso institucional: Los Planes de Pr«cticas. Tomo 1. VII Symposium Internacional sobre el Practicum. Practicum y Pr«cticas en empresas en la formaci\% universitaria. Espa.a: Universidad de Santiago de Compostela. 
Nieto Cano, J.M., \& Portela Pruaño, A. (2001). La cooperación entre agentes de innovación educativa. Formas y elementos básicos. Profesorado, revista de currículum y formación del profesorado, 5, 1-16.

Porto, M., \& Bolarín, M. J. (2013). Revisando las prácticas escolares: valoración de maestros-tutores. Profesorado, Revista de Currículum y Formación del Profesorado, 17(2), 461-477. 